\title{
The Bifunctional Enzyme, GenB4, Catalyzes the Last Step of Gentamicin 3',4'-Di-Deoxygenation via Reduction and Transamination Activities
}

\section{CURRENT STATUS: ACCEPTED}

Microbial Cell Factories $\triangle$ BMC

Xiaotang Chen

Shenyang Pharmaceutical University

376416460@qq.comCorresponding Author

ORCiD: https://orcid.org/0000-0003-0467-1488

Hui Zhang

Shenyang Pharmaceutical University

Shaotong Zhou

Shenyang Pharmaceutical University

Mingjun Bi

Shenyang Pharmaceutical University

Shizhou Qi

Shenyang Pharmaceutical University

Huiyuan Gao

Shenyang Pharmaceutical University

Xianpu Ni

Shenyang Pharmaceutical University

Huanzhang Xia

Shenyang Pharmaceutical University

DOI:

10.21203/rs.2.18758/v1

\section{SUBJECT AREAS}

Applied \& Industrial Microbiology

\section{KEYWORDS}

gentamicin, di-deoxygenation, GenB4, reduction activity, transamination activity 
Abstract

Background

New semi-synthetic aminoglycoside antibiotics generally use chemical modifications to avoid inactivity from pathogens. One of the most used modifications is $3^{\prime}, 4^{\prime}$-di-deoxygenation, which imitates the structure of gentamicin. However, the mechanism of di-deoxygenation has not been clearly elucidated.

\section{Results}

Here, we report that the bifunctional enzyme, GenB4, catalyzes the last step of gentamicin $3^{\prime}, 4^{\prime}$-dideoxygenation via reduction and transamination activities. Following disruption of genB4 in wildtypeM. echinospora, its products accumulated in 6'-deamino-6'-oxoverdamicin (1), verdamicin C2a (2), and its epimer, verdamicin C2 (3). Following disruption of genB4 in M. echinospora $\triangle$ genK, its products accumulated in sisomicin (4) and 6'-N-methylsisomicin (5, G-52). Following in vitro catalytic reactions, GenB4 transformed sisomicin (4) to gentamicin C1a (9) and transformed verdamicin C2a (2) and its epimer, verdamicin C2 (3), to gentamicin C2a (11) and gentamicin C2 (12), respectively. Conclusion

This finding indicated that in addition to its transamination activity, GenB4 exhibits specific 4', $5^{\prime}$ double-bond reducing activity and is responsible for the last step of gentamicin $3^{\prime}, 4^{\prime}$-dideoxygenation. Taken together, we propose three new intermediates that may refine and supplement the specific biosynthetic pathway of gentamicin C components and lay the foundation for the complete elucidation of di-deoxygenation mechanisms.

Full Text

Due to technical limitations, full-text HTML conversion of this manuscript could not be completed. However, the manuscript can be downloaded and accessed as a PDF.

Figures 


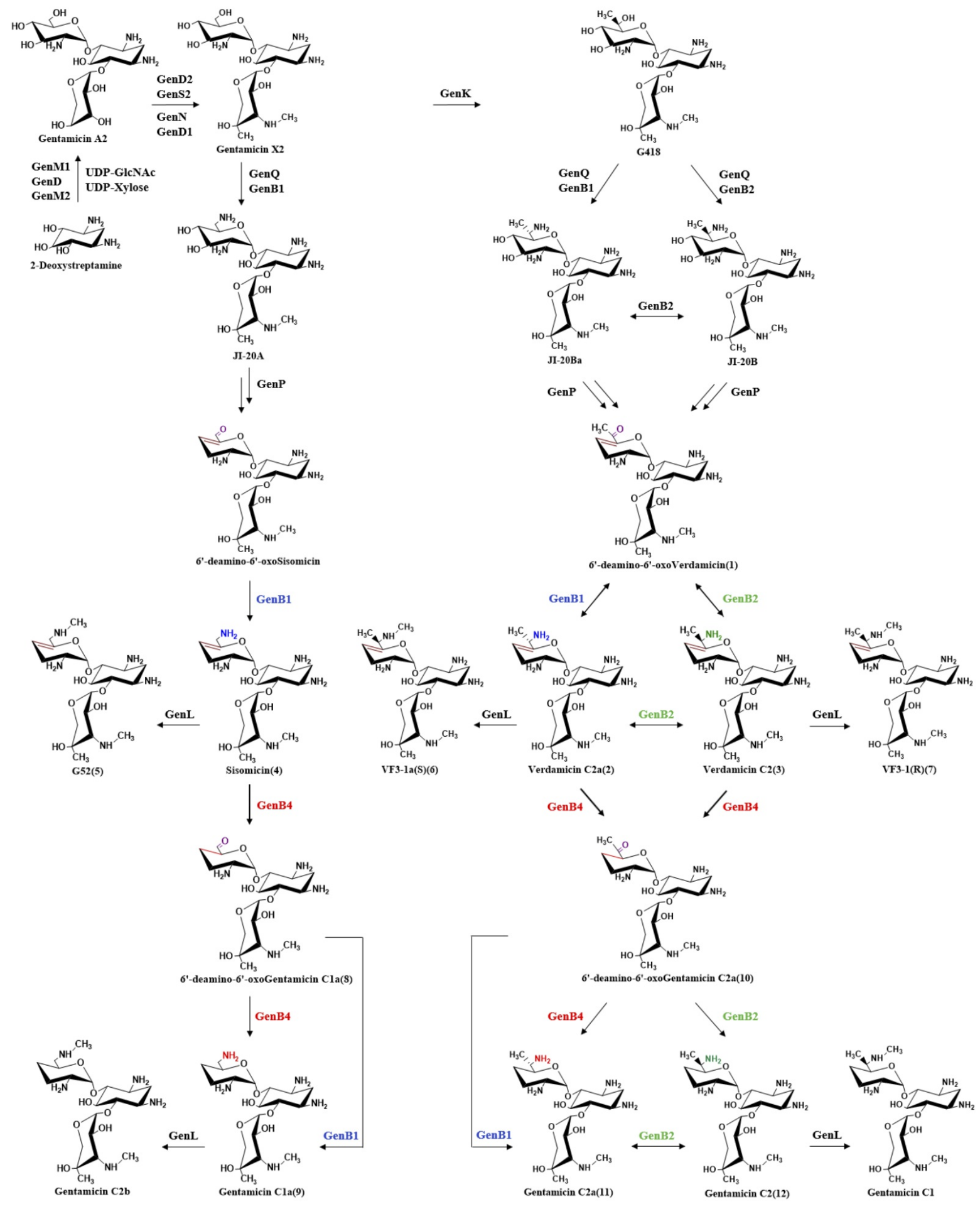

Figure 1

Biosynthetic pathway of gentamicin. 

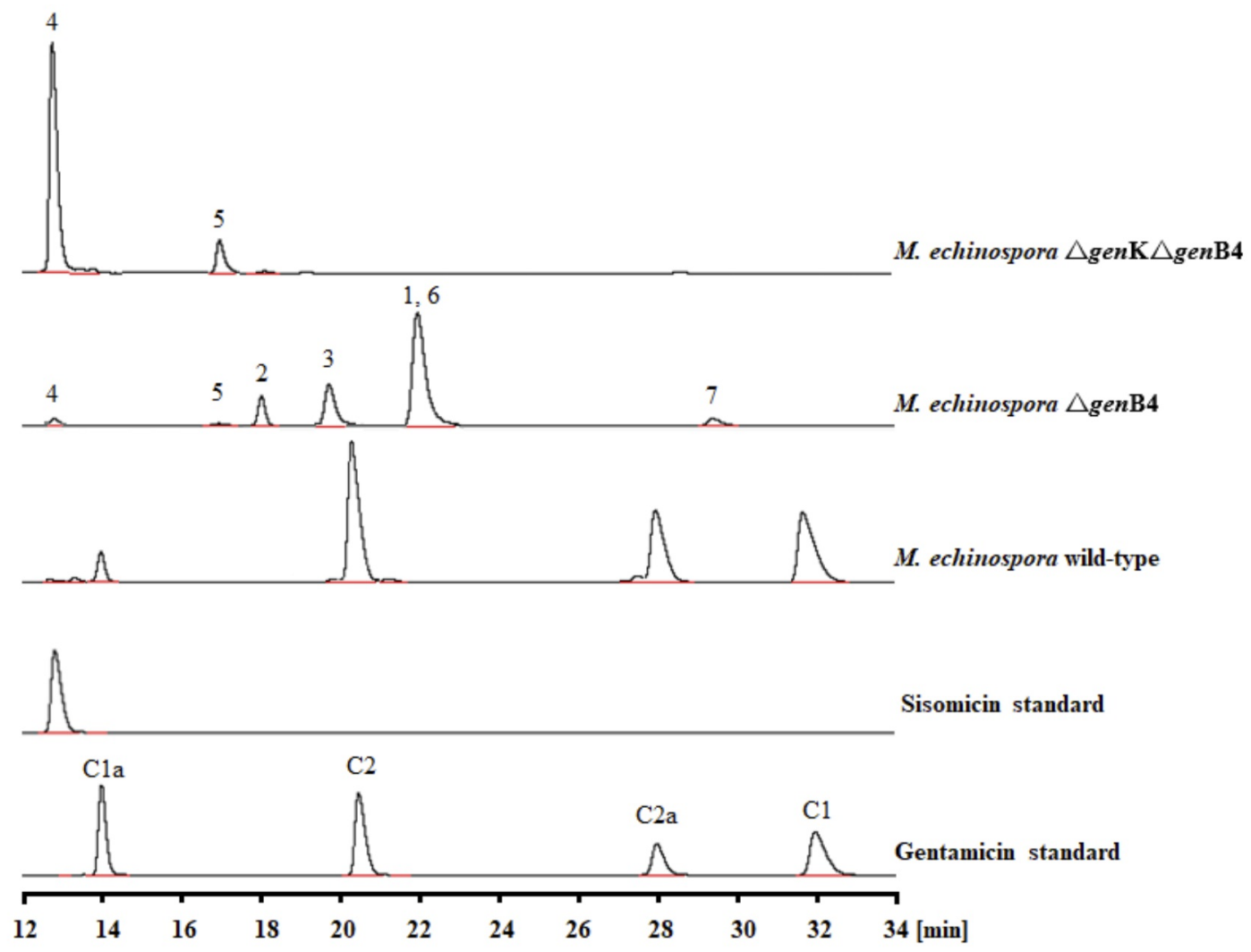

Figure 2

HPLC-ELSD analysis of fermentation production by M. echinospora wild type and mutants. 
a

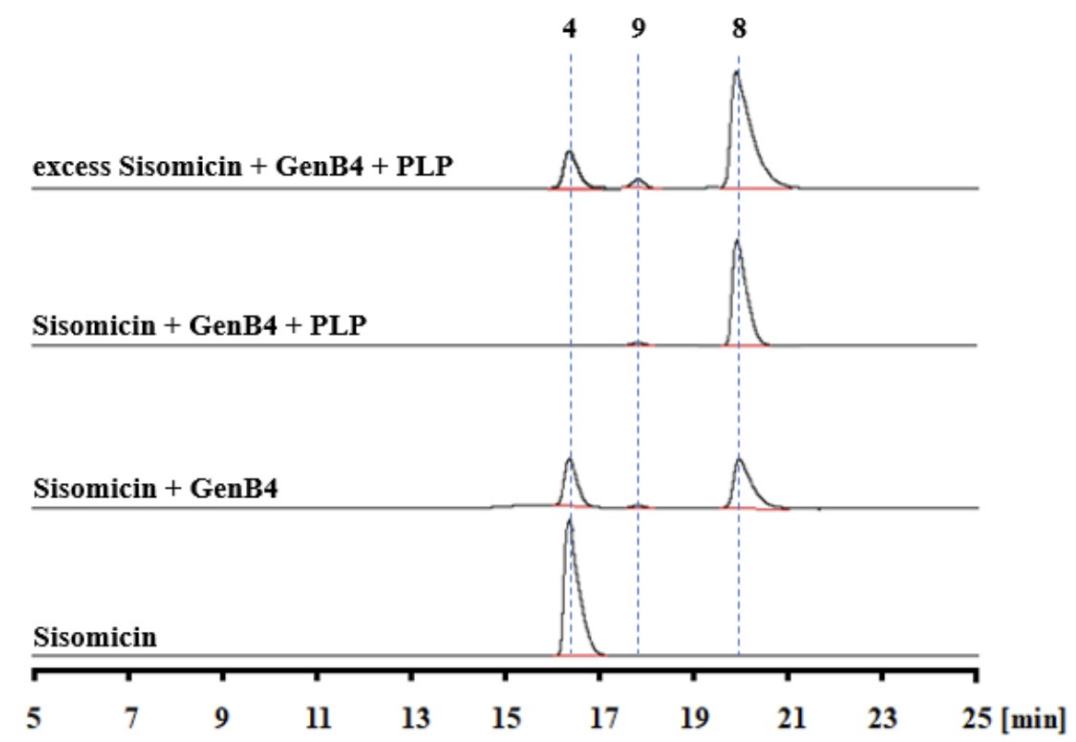

$\mathrm{b}$

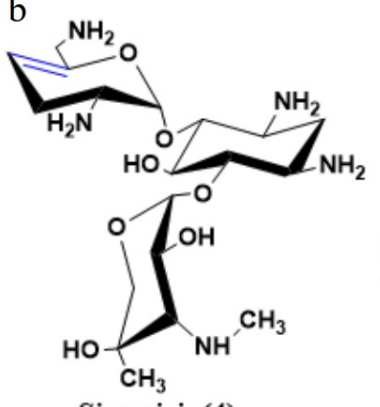

Sisomicin(4)

$\mathrm{m} / \mathbf{z}: 447.27$

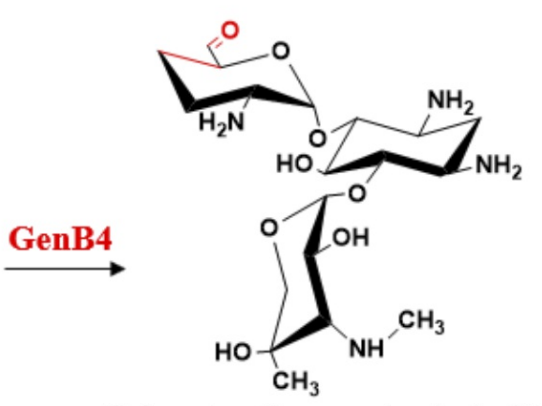

6'-deamino-6'-oxogentamincin $\mathrm{C} 1 \mathrm{a}(8)$

$\mathrm{m} / \mathrm{z}: \mathbf{4 4 8 . 2 5}$

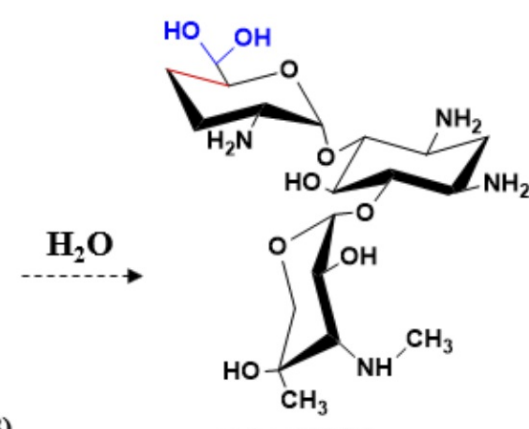

$\mathrm{m} / \mathrm{z}: 466.26$

c

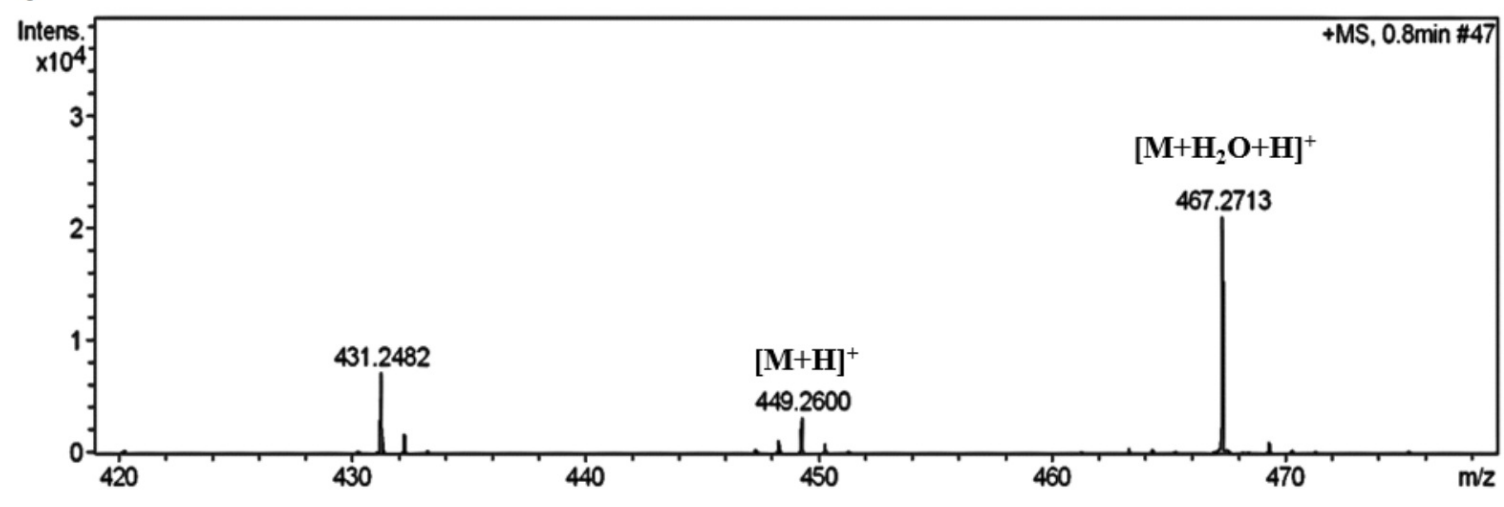

Figure 3

Analysis of GenB4-catalyzed reactions. a) HPLC-ELSD analysis of GenB4 enzymatic assays.

b) Proposed structure of the catalysate. c) Mass spectra analysis of compound (8). $[\mathrm{M}+\mathrm{H}]+$ $m / z$ 449, $[\mathrm{M}+\mathrm{H} 2 \mathrm{O}+\mathrm{H}]+\mathrm{m} / \mathrm{z} 467$. 
a
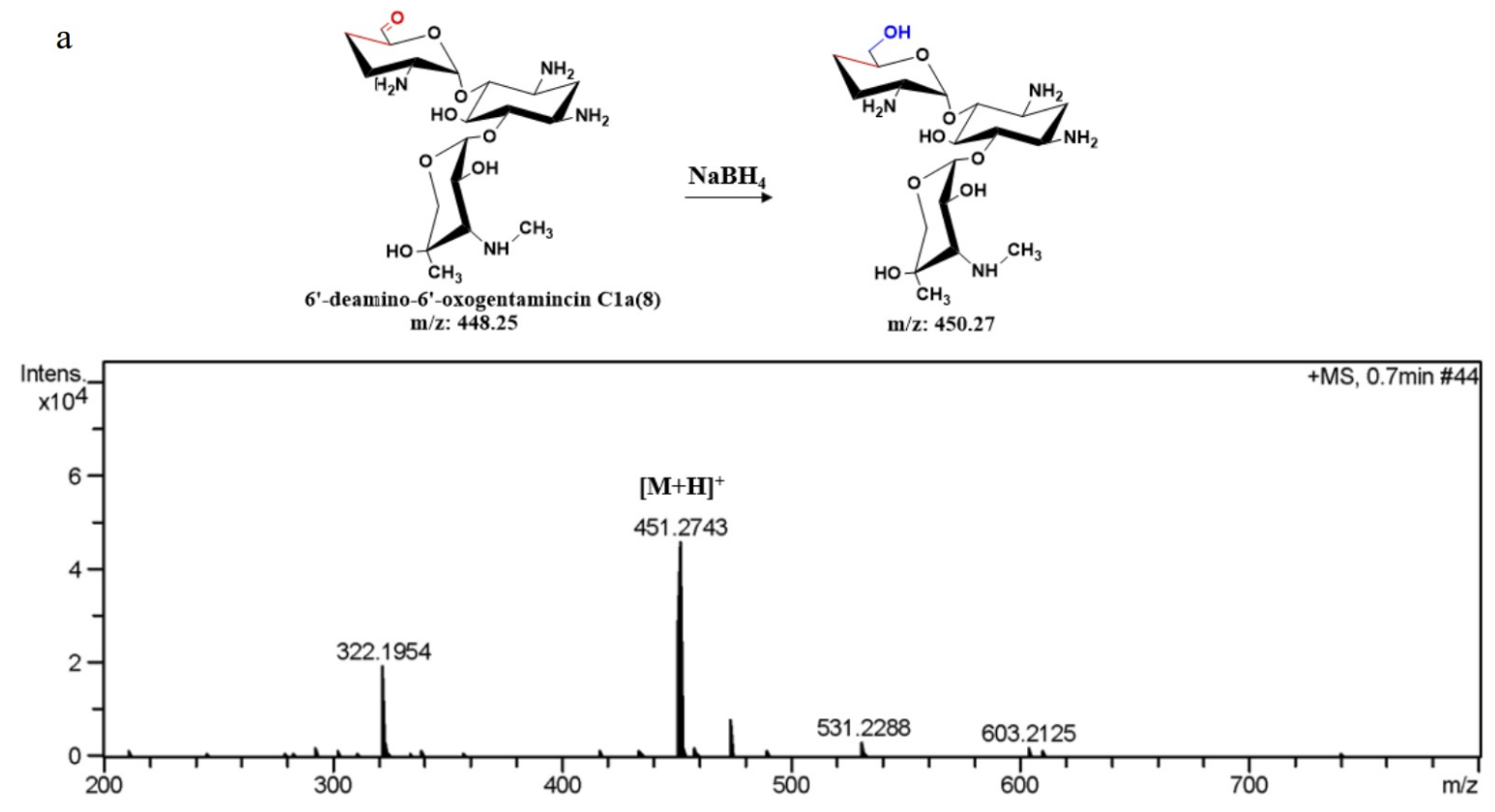

b
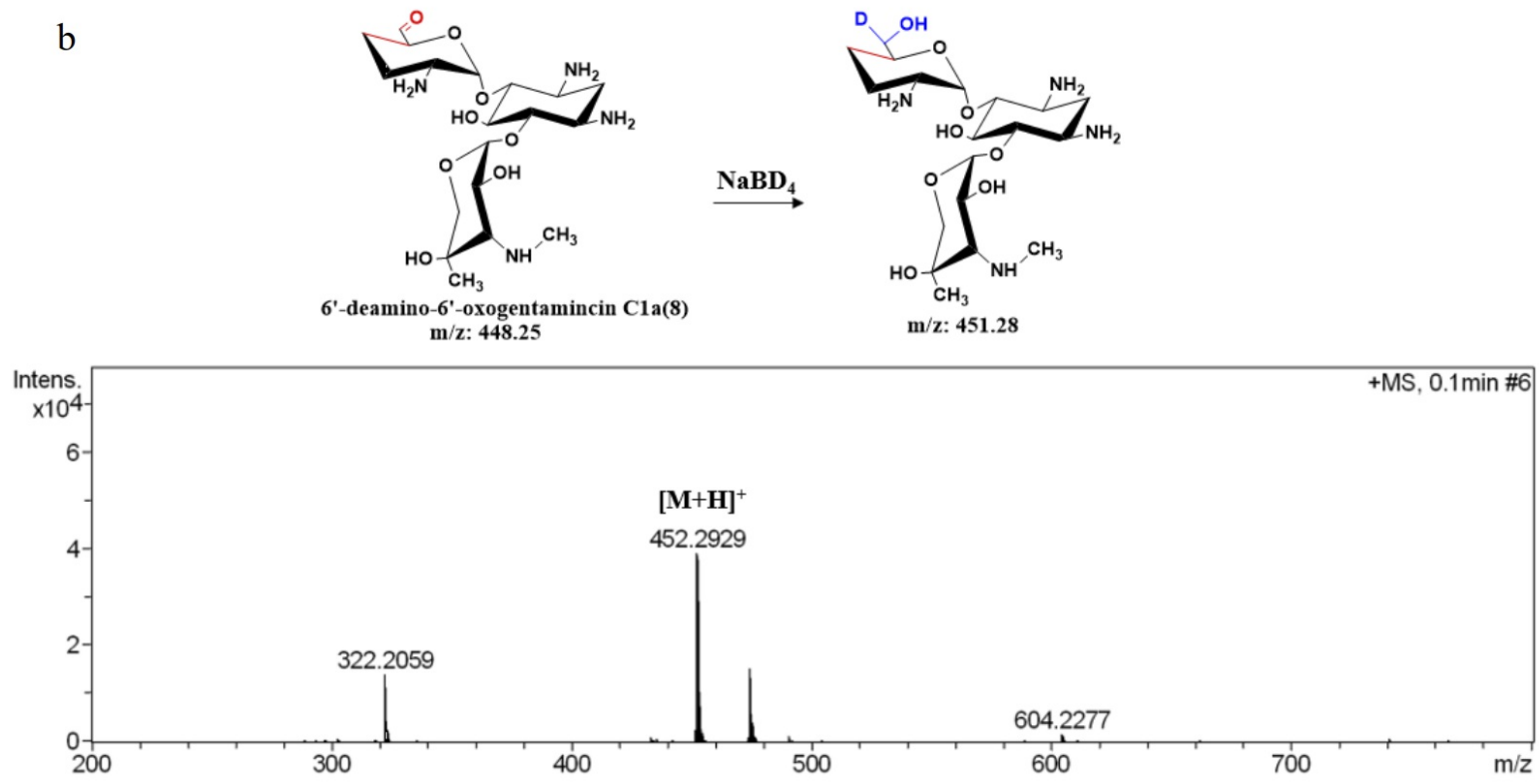

Figure 4

Mass-spectra analyses of compound (8) reduced with $\mathrm{NaBH} 4$ or NaBD4. a)Mass-spectra analysis of compound (8) reduced with $\mathrm{NaBH} 4$. b) Mass-spectra analysis of compound (8) reduced with NaBD4.

a

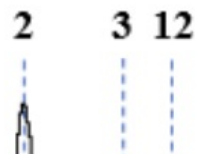

1011

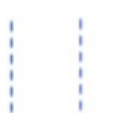



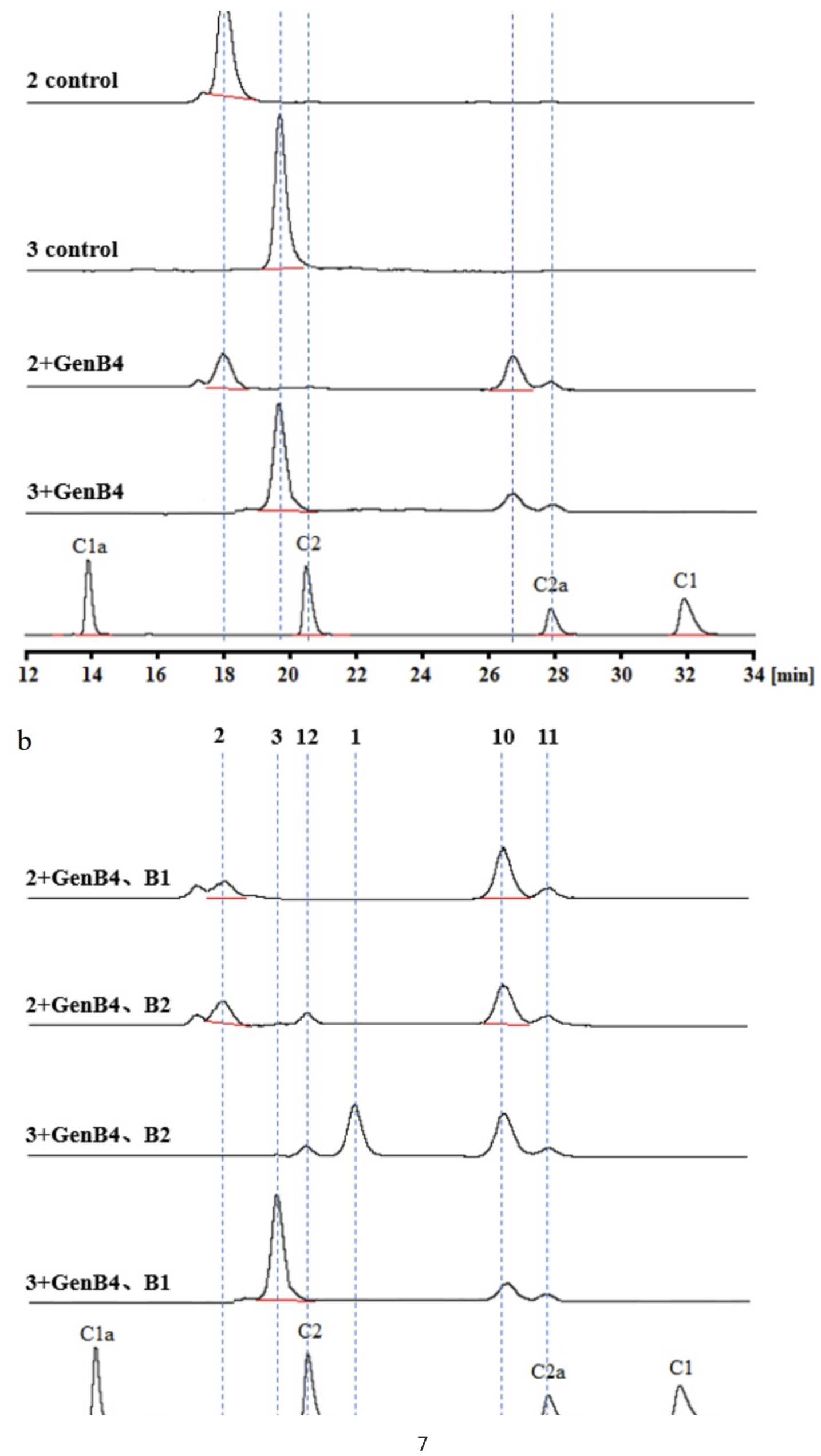


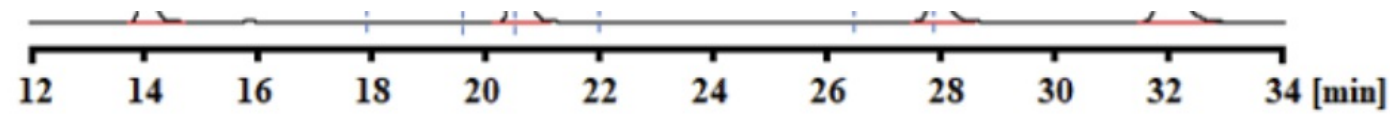

Figure 5

HPLC-ELSD analysis of GenB4-catalyzed verdamicin C2a (2) and verdamicin C2 (3) to gentamicin C2a (11) and gentamicin C2 (12).

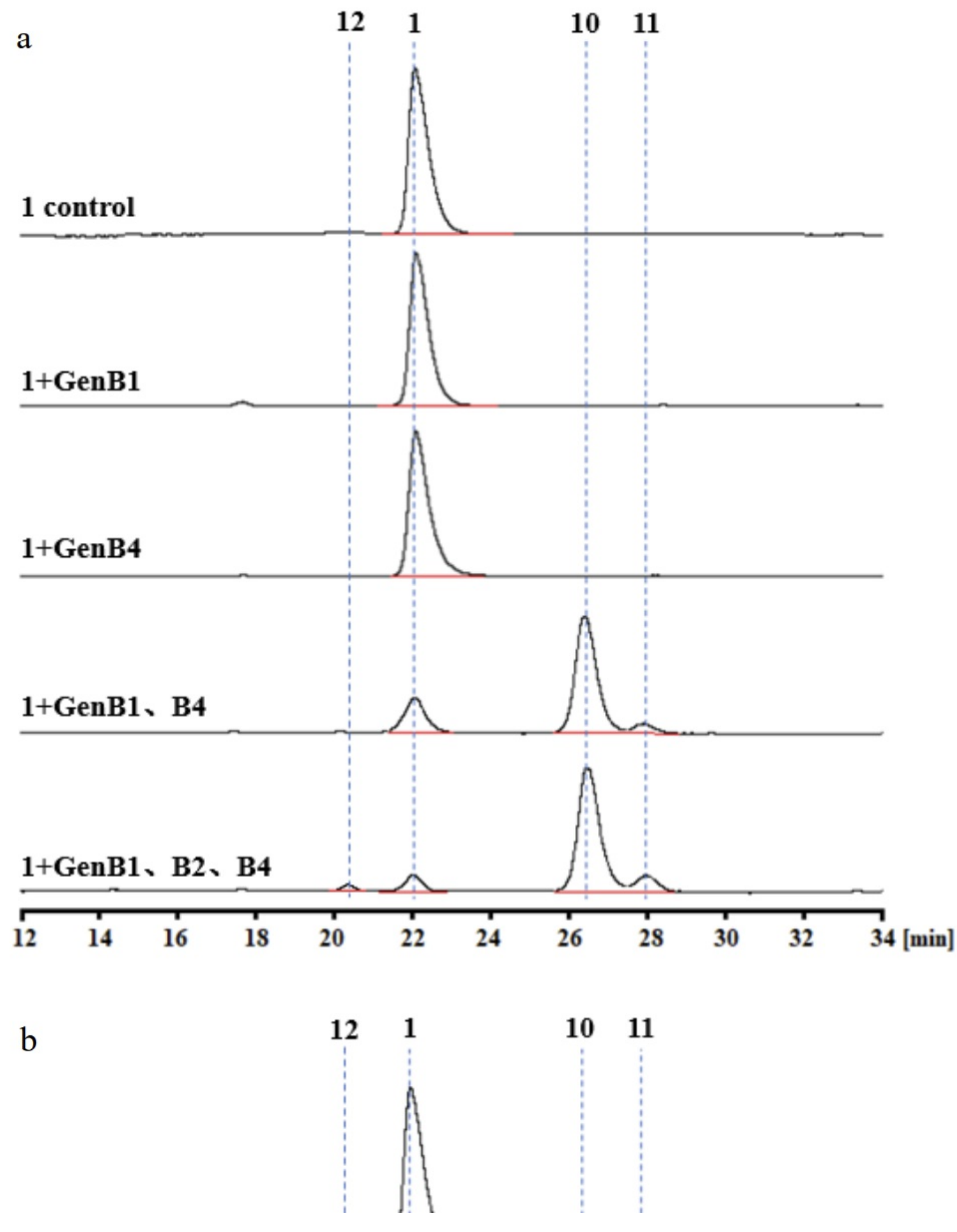




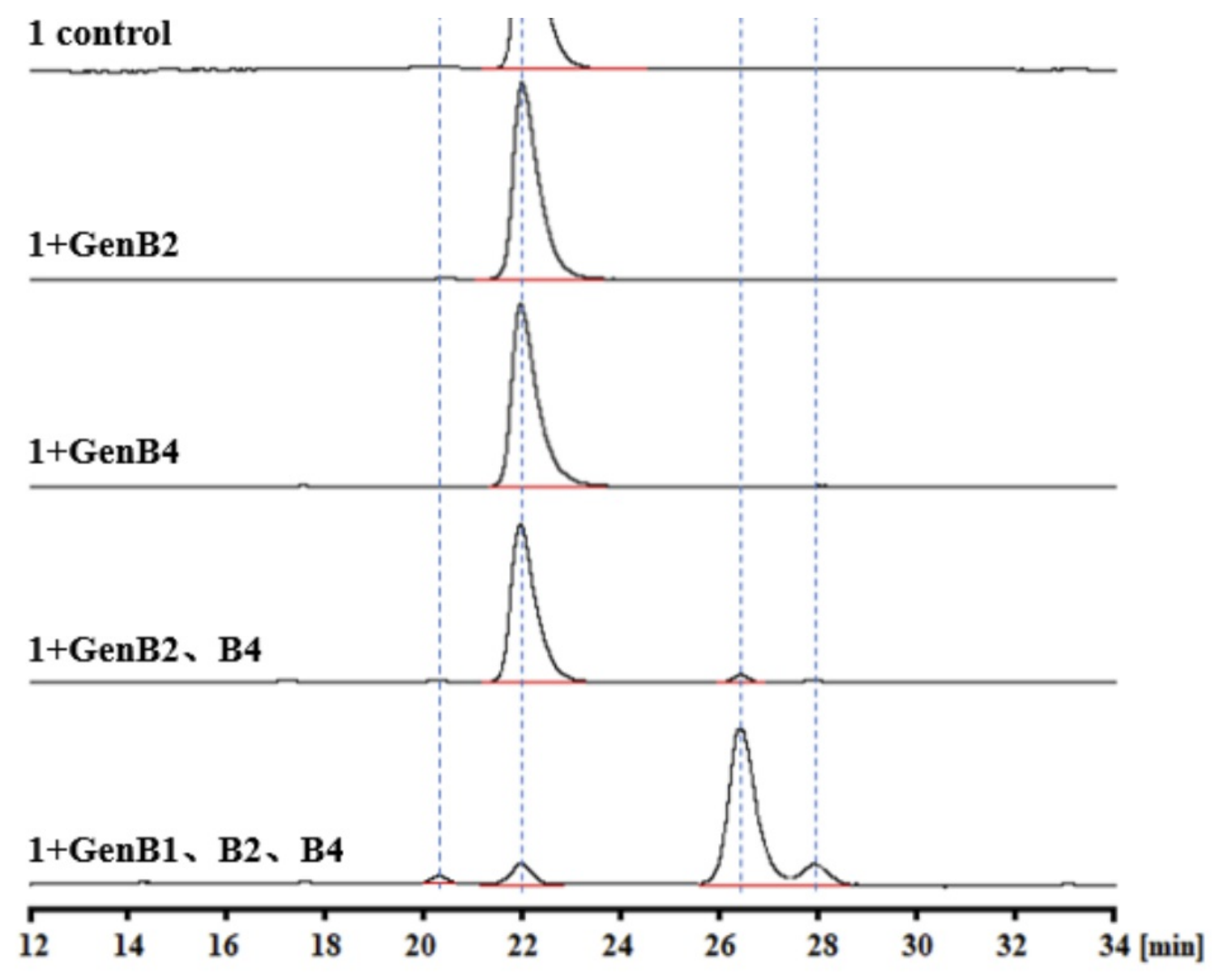

Figure 6

HPLC-ELSD analysis of 6'-deamino-6'-oxoverdamicin (1) catalyzed by GenB4.

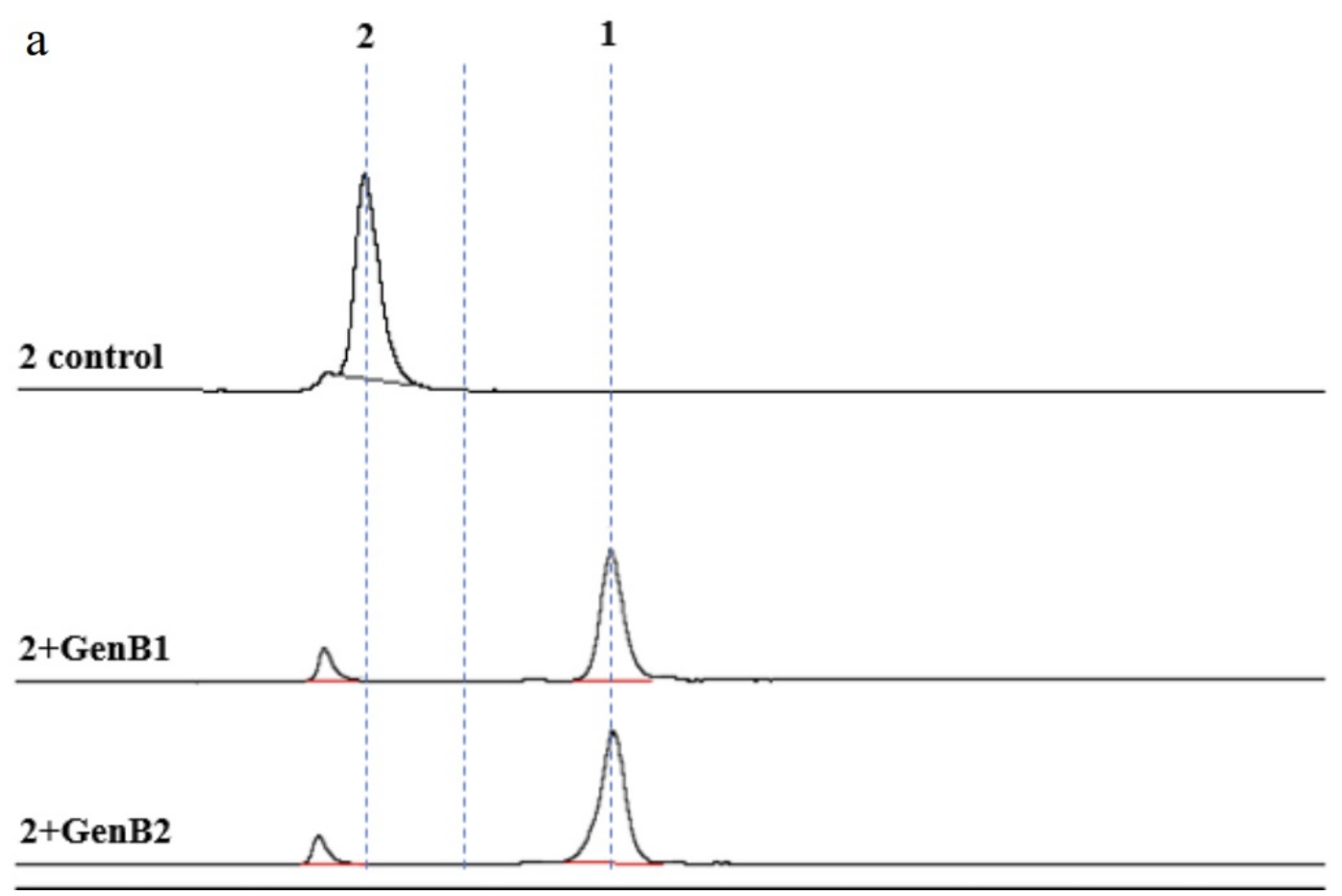




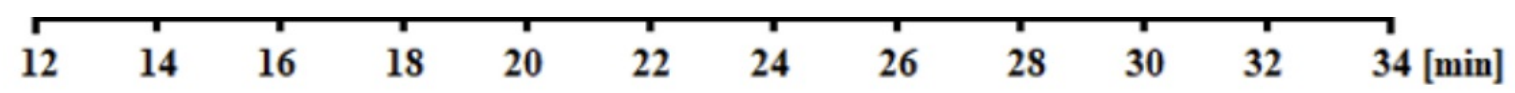

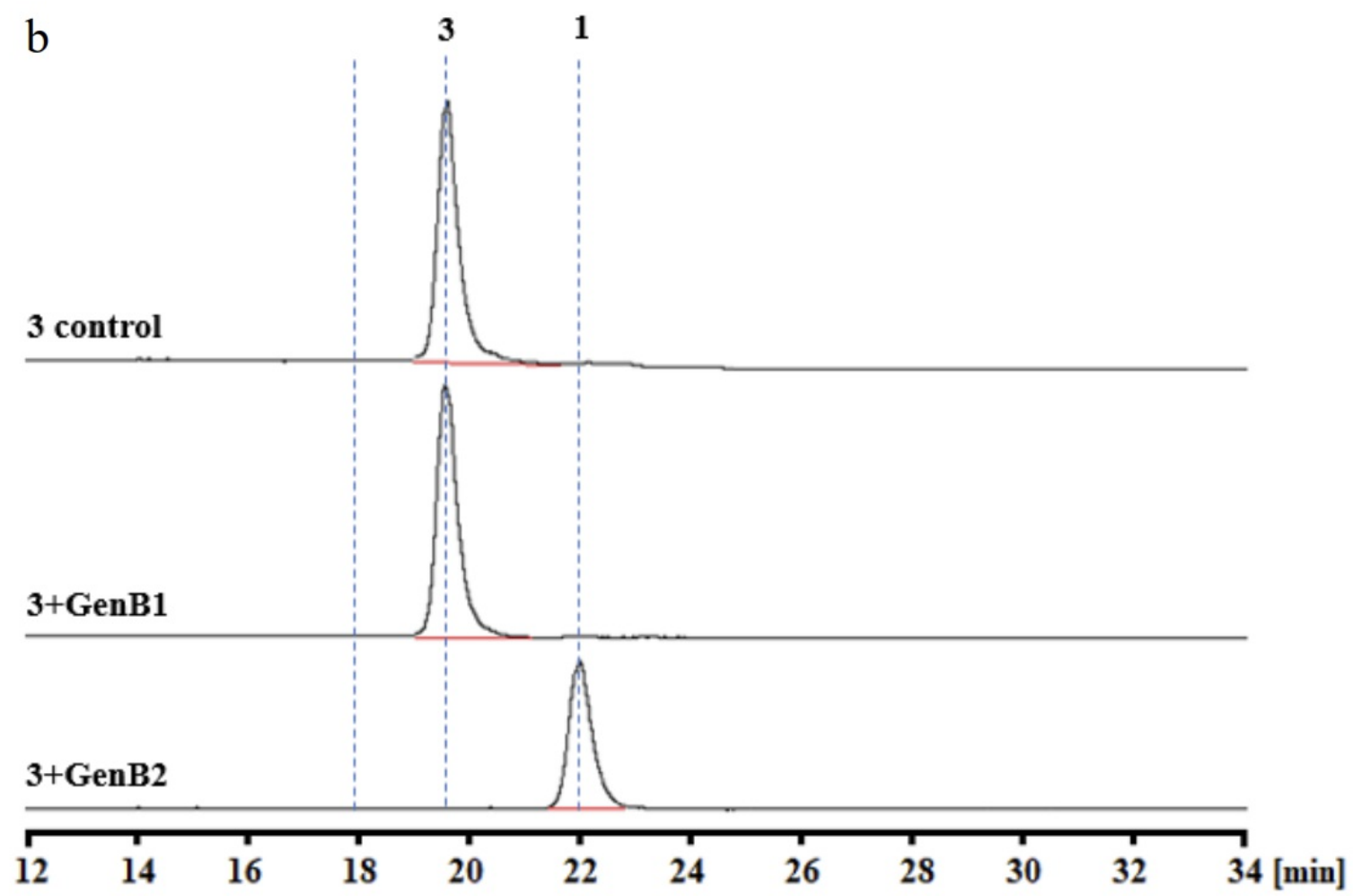

Figure 7

HPLC-ELSD analysis of the transformations of (1), (2), and (3).

\section{Supplementary Files}

This is a list of supplementary files associated with this preprint. Click to download.

fig S10.pdf

fig S13.pdf

fig S5.pdf

fig S6.pdf

fig S12.pdf

fig S11.pdf

fig S9.pdf

fig S8.pdf

fig S3.pdf

fig S4.pdf

table S1.pdf

fig 57.pdf

fig S2.pdf

fig S1.pdf 
